\title{
An Assessment of the Social Cognitive Predictors of Exclusive Breastfeeding Behavior Using the Health Action Process Approach
}

\begin{abstract}
Rationale: In the United States rates of exclusive breastfeeding duration remain exceedingly low. Exclusive breastfeeding is a complex learned behavior that is influenced by social cognitive, interpersonal, and structural factors. Interventions are needed that address factors at multiple levels of the social-ecological model. This study was designed to examine the social cognitive predictors of exclusive breastfeeding behavior in a sample of low-income women attending the Special Supplemental Nutrition Program for Women, Infants, and Children (WIC) breastfeeding peer counseling program and enrolled in the Lactation Advice Through Texting Can Help (LATCH) study.
\end{abstract}

Objectives: The objectives were to examine whether: (1) the theoretical model, the Health Action Process Approach (HAPA), fit the data well; (2) planning mediated the effect of intentions and maintenance self-efficacy on exclusive breastfeeding; and (3) recovery selfefficacy mediated the association between maintenance self-efficacy and exclusive breastfeeding behavior.

Methods: Outcome expectancies, action self-efficacy and intentions were assessed prenatally at baseline in $\mathrm{N}=119$ participants. Maintenance self-efficacy, planning, recovery self-efficacy and breastfeeding behavior were measured at two weeks post partum. Structural equation modeling with mean and variance adjusted Weighted Least Squares estimation was used to examine the applicability of the HAPA model to the data.

Results: Phase specific self-efficacy and planning significantly predicted exclusive breastfeeding status. Planning and recovery self-efficacy mediated the association between maintenance self- 
efficacy and exclusive breastfeeding. Planning did not emerge as a mediator between intentions and behavior.

Conclusion: These results demonstrate the utility of the HAPA model in predicting exclusive breastfeeding behavior among low-income women attending WIC. LATCH is a theoretically sound text messaging intervention that can be used to augment and reinforce the WIC breastfeeding peer counseling process.

\section{Key Words}

Exclusive breastfeeding, breastfeeding, behavioral theory, WIC, mHealth, text messaging, USA 


\section{Background}

Breastfeeding rates in the United States (U.S.) remain exceedingly low despite the many known short and long term benefits for both mother and baby. As of 2011, 81.1\% of mothers in the U.S. were initiating breastfeeding yet just $44.4 \%$ of infants were exclusively breastfed to 3 months of age and only $22.3 \%$ to six months of age (Centers for Disease Control and Prevention (CDC), 2016). These rates remain below the Healthy People 2020 objectives of $46.2 \%$ and $25.5 \%$ exclusively breastfeeding to 3 and 6 months of age, respectively (Healthy People 2020, 2015). The Special Supplemental Nutrition Program for Women Infants and Children (WIC) serves 53.0\% of all infants nationally (United States Department of Agriculture, 2015); however, rates of breastfeeding initiation and duration are dramatically lower among women who attend WIC than among women who do not attend WIC, regardless of their socioeconomic status (Jensen, 2012; Ryan \& Zhou, 2006).

Breastfeeding is a complex learned behavior influenced by factors at multiple levels of the social-ecological model. The motivation to breastfeed and breastfeeding behavior are influenced by social cognitive factors such as risk perception, outcome expectancies, intentions, self-efficacy and planning (de Jager et al., 2013). Lactation education, management and support can also affect a mothers' decision to breastfeed and her potential for long-term continuation (Rollins et al., 2016). Social and family support as well as societal norms and pressures (including the marketing of breast milk substitutes) can influence breastfeeding motivation and behavior (Rollins et al., 2016). The larger policy environment also affects employment and childcare conditions that can either promote or hinder breastfeeding behavior (Rollins et al., 2016). Community-based breastfeeding promotion and support, such as breastfeeding peer counseling, is a key aspect of successful strategies designed to improve breastfeeding outcomes (Chapman et al., 2010; Perez-Escamilla, 2012; WHO/UNICEF, 2003). In fact, a recent 
systematic review indicated that interventions combining health systems and community-based services (e.g., breastfeeding peer counseling) demonstrate the largest effects on breastfeeding outcomes (Rollins et al., 2016). Furthermore, several case studies have demonstrated that behavior change communication should be an integral part of any large scale breastfeeding promotion and support strategy (Martin et al., 2010).

This paper describes the social cognitive results of the Lactation Advice Through Texting Can Help (LATCH) study. LATCH was a multi-site single-blind randomized controlled trial designed to test the effectiveness of a two-way text messaging intervention encouraging exclusive breastfeeding among low-income women enrolled in WIC. It was the first of its kind to combine in-person prenatal and post partum breastfeeding education and support (breastfeeding peer counseling) with mHealth technology (text messaging). The effectiveness of Breastfeeding Peer Counseling (BFPC) has been demonstrated through multiple randomized controlled trials (Anderson et al., 2005; Chapman et al., 2004a; Chapman et al., 2004b) and summarized through literature reviews (Chapman et al., 2010; Chapman \& Perez-Escamilla, 2012), thus the text messages were designed to augment and reinforce the BFPC process, not replace it.

\section{Theoretical framework for the present study}

The LATCH study was based upon the Health Action Process Approach (HAPA) to behavior change (Schwarzer, 2008) which is described below in the context of breastfeeding behavior. The HAPA posits that there are two essential phases to behavior change (Figure 1). In the motivational phase, three factors influence the formation of an intention to breastfeed. Confidence in one's ability to initiate breastfeeding (action self-efficacy), behavioral expectations that help a mother weigh the pro's and con's of breastfeeding (outcome expectancies), and beliefs about how risky breastfeeding may be (risk-perception) together 
predict the intention to breastfeed in the motivational stage (Schwarzer, 2008). The volitional or post-intentional stage begins once baby is born and breastfeeding has been initiated. Here, action and coping planning are hypothesized to mediate the association between intentions and behavior (Schwarzer, 2008). In other words, once an intention has been formed it has to be translated into action though the delineation of specific steps - the "when, where, and how" of initiating the behavior (action plans), as well as the identification of anticipated barriers and setbacks with corresponding coping plans to address them (Schwarzer, 2014). Situational factors and unanticipated barriers and setbacks that inevitably arise can be planned for by the establishment of a strong social support network (Schwarzer, 2014). In this way, the intention to breastfeed is broken down into more immediate goals and plans that serve to achieve the breastfeeding behavior (Schwarzer, 2014).

\section{[INSERT FIGURE 1 HERE]}

Phase-specific self-efficacy (i.e., action, maintenance, and recovery self-efficacy) constitutes optimistic beliefs in one's ability to set and pursue health goals (Renner et al., 2008; Schwarzer \& Renner, 2000; Schwarzer et al., 2007) and plays an essential role in breastfeeding behavior. Maintenance self-efficacy refers to confidence in one's capability to continue (exclusively) breastfeeding despite barriers and setbacks and recovery self-efficacy refers to confidence in one's capability to resume breastfeeding even after an interruption or after having stopped (Schwarzer, 2008). Importantly, an individual can simultaneously hold different types and different levels of phase-specific self-efficacy (Renner et al., 2008; Schwarzer \& Renner, 2000; Schwarzer et al., 2007). For example, a mother may have high confidence in her ability to initiate breastfeeding once baby is born, but less confidence in her ability to continue breastfeeding in the face of barriers or setbacks like engorgement, plugged ducts, and nipple 
pain. It should be noted that each of the constructs of the HAPA model can be mapped to the behavior change technique (BCT) taxonomy (Michie et al, 2013). The BCT taxonomic clusters that apply to the HAPA model include: (1) pros and cons and comparative imagining of future outcomes (for the outcome expectancies and risk perception constructs, respectively); (2) verbal persuasion to boost self-efficacy (for the action, maintenance, and recovery self-efficacy constructs); and (3) action planning (including implementation intentions) and problem solving/coping planning (for the action and coping planning constructs, respectively).

The HAPA has proven effective in disease prevention interventions targeting such varied behaviors as dental flossing, seat belt use, physical activity, smoking cessation, breast selfexamination, and healthy dietary behaviors (A. Luszczynska, 2004; Renner et al., 2008; Schwarzer et al., 2007; Schwarzer R., 2008). The present study is the first in which it was applied to a breastfeeding promotion and support intervention. The purpose of this study was to examine whether the social cognitive constructs of the HAPA model predicted exclusive breastfeeding $(\mathrm{EBF})$ behavior at two weeks post partum in a sample of WIC participants. Specifically, we examined whether: (1) the theoretical HAPA model fit the data well; (2) planning mediated the effect of intentions and maintenance self-efficacy on EBF; and (3) recovery self-efficacy mediated the association between maintenance self-efficacy and EBF behavior.

\section{Methods}

Study Design

The LATCH study (parent study) and the present study were approved by the Yale University Human Subjects Investigation Committee. LATCH was conducted from August, 2014 to January, 2016 and was registered at clinicaltrials.gov (protocol \#: 1206010472) prior to the start of recruitment. 
Study population. Eligible participants were pregnant women 18 years of age or older who attended the breastfeeding peer counseling program at one of the four study sites, were 28 weeks gestation or less, expressed the intention to breastfeed, conceived a singleton, and who had unlimited text messaging on their mobile phone. Women were excluded from the study if they had a miscarriage, gave birth prematurely ( $<37$ weeks gestation), withdrew from the BFPC program, or were carrying multiples. Participants were excluded once their baby was born if the baby weighed less than 5 pounds, spent more than 3 days in the neonatal intensive care unit, or if medication given to mother or baby precluded breastfeeding.

Study sites and staff. Four WIC BFPC sites in Connecticut participated in the study representing a teaching hospital, a federally qualified health center, and two community-based agencies all serving low-income and minority women. A total of seven BFPC's and three Internationally Board Certified Lactation Consultants (IBCLC's) agreed to participate and were trained on the LATCH study protocol in a half-day training in May of 2014. Booster trainings were provided on an as-needed basis thereafter. Four of the seven BFPC's were part-time and one IBCLC was part time at two different study sites.

Text messaging platform. Text messages were sent to intervention participants through the HIPAA-compliant Mobile Commons (MC) two-way text messaging platform. Messages designed for each trimester of pregnancy and up to three months postpartum were sent automatically by the MC platform using a pre-determined schedule. The platform recorded when the messages were sent, whether they were received by the participant, and all message exchanges between participants and their BFPC.

Message content, design, and frequency. Prenatal and peripartum text messages were developed during the pilot study (Harari, 2014) and expanded upon to cover up to three months 
postpartum for the current study. Message content covered the benefits of breastfeeding for mother and child, debunked breastfeeding myths, showed examples of proper positioning, explained how to tell if baby is getting enough milk, and reinforced the BFPC's supportive role. Messages were developed with feedback from BFPC's and IBCLC's. Next, messages were mapped to the constructs of the HAPA model through an iterative process with Co-PIs (RPE \& $\mathrm{NH})$. This process was designed to ensure that a relatively equal number of text messages addressed the core constructs of the HAPA model (i.e., self-efficacy and planning). Messages were designed to address outcome expectancies, action self-efficacy and action planning during the prenatal period and maintenance, recovery self-efficacy, and coping planning in the post partum period. Self-efficacy messages were designed to improve participants' confidence in their capability to initiate breastfeeding (action), continue breastfeeding in the face of barriers and setbacks (maintenance), and begin breastfeeding again if they have stopped (recovery). Selfefficacy is acquired through indirect or vicarious experience, verbal persuasion, and direct or mastery experience (Bandura, 1977). During the prenatal period messages focused on acquiring self-efficacy through indirect or vicarious experience (e.g., videos and photos of proper positioning) and/or verbal persuasion (e.g., text message conversations with BFPC). In the postpartum period self-efficacy was reinforced through direct or mastery experience and verbal persuasion. Table 1 contains examples of the text messages designed to address these constructs.

\section{[INSERT TABLE 1 HERE]}

Messages were also "gain" framed - emphasizing the positive aspects of engaging in the behavior (Rothman \& Salovey, 1997; Tversky \& Kahneman, 1981). Any changes to message content based on the message framing exercise were reviewed by IBCLC's for clarity. Text messages were translated into Spanish and content for multimedia messages was obtained in 
Spanish as well. Finally, messages were tailored, addressing each participant by her first name and were periodically signed with the peer counselor's first name.

In addition to the standard of care breastfeeding peer counseling program (described below), intervention participants received a series of text messages during the prenatal and post partum period sent according to a predetermined schedule. The schedule, tested and established with feedback from participants during the pilot phase, varied message frequency over time. Text messaging was initiated by researchers with the first text sent automatically after intake from the MC platform.

Procedure. Participants were recruited, consented, and enrolled in LATCH by their BFPC, at which point they received an automated text message from the MC platform, indicating that they had been enrolled. Participants were asked to reply "yes" to confirm their enrollment. Next, each was called by a bilingual bicultural research assistant trained in conducting telephone interviews. Participants were asked to complete their baseline interview over the phone and upon completion were randomized to the intervention or control group. Those in the control arm received the standard of care WIC Loving Support breastfeeding peer counseling program (Epstein, 2015). The Loving Support model relies on breastfeeding peer counselors who are members of the target population for a given WIC clinic and have breastfed previously, thus they serve as breastfeeding role models to WIC participants (Epstein, 2015). BFPC's are trained to provide breastfeeding education and support through one-on-one counseling which can occur over the phone or in-person. Supervision and support is provided by an IBCLC who is also onsite. Participants in the intervention arm received the standard of care BFPC program + the SMS intervention. 
The peer counselors in the LATCH study used the MC platform to monitor and interact with intervention participants through text prenatally and up to two weeks postpartum. By study design, control group participants did not exchange text messages with their peer counselors via MC and were instructed not to text their BFPC's via their personal cell phones. Follow-up interviews were conducted by a trained bilingual bicultural interviewer who was blind to study condition at approximately 2 weeks post partum. Participants received $\$ 10$ for their baseline interview and $\$ 10$ for their 2 week interview follow-up interview.

\section{Measures}

Outcome variable. The dependent variable of interest was exclusive breastfeeding status (EBF vs. partial breastfeeding) at 2 weeks postpartum. The World Health Organization (WHO) defines EBF as an infant's consumption of breast milk only, no other liquids (not even water), allowing only for token amounts of medical fluids (WHO, 2015). Partial breastfeeding is defined as an infant's consumption of breast milk and formula, but no solids (Labbok \& Krasovec, 1990). To assess breastfeeding status we used a modified version of the breastfeeding status question from the Infant Feeding Practices Study II (IFPS II) neonatal questionnaire (CDC, 2014). Participants were asked to reply Yes or No to each of the following: "Over the past 24 hours, did you give your baby: (1) Breast milk; (2) Formula; (3) Water; (4) Tea; (5) Juice; (6) Other." Respondents were then categorized as exclusively, partially, or not breastfeeding based on the WHO definition.

Independent variables. All HAPA-related items were adapted from Schwarzer, 2008 and were measured on a four point scale: (1) very false, (2) somewhat false, (3) somewhat true, and (4) very true. All items were tested in a pilot study in the target population for understanding and difficulty. Each of the constructs and their corresponding indicator variables can be found in 
Table 3. Ranges, means, standard deviations and standardized factor loadings for each indicator are presented, along with Cronbach's alphas for each of the scales.

Outcome expectancies, action self-efficacy, and intentions were measured at time 1 . Intention to both initiate and EBF was measured using a modified version of the Infant Feeding Intentions scale (Nommsen-Rivers et al., 2010; Nommsen-Rivers \& Dewey, 2009). Action and coping planning, maintenance and recovery self-efficacy were measured at time 2 . It is important to note that at two weeks post partum EBF mothers were asked about maintaining/recovering their exclusive breastfeeding status while partially breastfeeding mothers were asked about maintaining their partial breastfeeding status and recovering their exclusive breastfeeding status.

Maternal biomedical characteristics. Other important descriptive characteristics measured at time 1 included the participant's age at recruitment, pre-pregnancy weight and height used to calculate Body Mass Index (BMI), baby's gestational age at recruitment, and whether the participant was primiparous or not. Variables measured at time 2 included the selfreported number of days post partum the participant experienced onset of lactation (copious milk production or lactogenesis II), and the baby's age at the time the two week follow-up interview was completed (in days).

Breastfeeding planning and experience. At baseline participants were asked to report whether they planned to exclusively breastfeed (yes/no) and partially breastfeed (yes/no) and the number of months of each. Previous breastfeeding experience (yes/no), was also recorded.

Demographic characteristics. Ethnicity (Hispanic/non-Hispanic), marital status (single never married, married, divorced/separated/widowed), living with partner (yes/no), maternal education level (high school grad/GED, < high school, > high school), and language preference (English/Spanish) were collected at time 1. 
Cell phone \& plan. Plan type (month-to-month or contract), type of phone (Smartphone or not), and data plan (unlimited or not) were also recorded.

Income and food insecurity. Finally, participants were asked to report their income level $(\leq \$ 1,000 / \mathrm{mo}, \$ 1,000-\$ 2,000+/ \mathrm{mo}$, or Don't Know/Refused) and whether they were enrolled in the Supplemental Nutrition Assistance Program (SNAP; yes/no).

Analyses

In order to determine whether the constructs were related to one another and to EBF behavior, structural equation modeling with mean and variance adjusted Weighted Least Squares (WLSMV) estimation was performed using MPlus version 7.2 (Muthén, 1998 - 2009). The pairwise deletion method was used for the treatment of missing values (Muthén, 1998 - 2009). Evaluation of model fit was based on the Comparative Fit Index (CFI), Tucker-Lewis Fit Index (TLI), and the Root Mean Square Error of Approximation (RMSEA) in conjunction with an examination of the parameter estimates and the direction and magnitude of the correlations between latent factors. The following cutoffs were used for acceptable fit: CFI $\geq 0.96, T L I \geq$ 0.96, and RMSEA $<0.06$ (Schreiber et al., 2006).

In step 1 confirmatory factor analysis was used to develop an unconstrained measurement model with acceptable fit to the data based on the HAPA (Figure 1). The inclusion criteria specified that women enrolled in the study had to intend to breastfeed. Thus, the latent construct for risk perception was omitted from the measurement model because it is considered a distal antecedent to intentions. Since risk perception is important early in the motivational phase, before an intention is formed, it was not considered as important of an explanatory construct as action self-efficacy and outcome expectancies, two more proximal determinants of intentions. It 
was also determined in this step that action and coping planning should be modeled as a single latent planning construct because of the presence of multicollinearity between several indicators.

In step 2, the measurement model was modified to become a structural equation model representing the HAPA. The recursive hypothetical model consisted of six latent variables. All latent variables used multiple indicators as shown in Table 3. A structural model was tested whereby action self-efficacy and outcome expectancies were specified as exogenous latent variables and predictors of behavioral intention. Intention and maintenance self-efficacy were specified as predictors of planning; planning, maintenance self-efficacy and recovery-selfefficacy were specified as predictors of EBF behavior. The Dropout analysis revealed statistically significant differences between study participants $(\mathrm{N}=119)$ and those who dropped out $(\mathrm{N}=47)$ with respect to three baseline variables: Planned partial BF duration (mo.'s), maternal education, and language preference. Compared to study participants, those who were lost to follow-up planned to partially breastfeed for a longer duration, were more likely not to have graduated high school, and were more likely to speak English. Planned partial BF duration was the only baseline variable that significantly impacted the parameter estimates of the SEM. Thus, the structural model controlled for this variable as well as onset of lactation (number of days post partum). Both of these variables were also significantly associated with EBF status at two weeks post partum (Table 2).

\section{Sample}

Figure 2 depicts the sample selection for these analyses which were limited to participants who were still breastfeeding at time 2. Eight of the 127 participants with outcome data at two weeks post partum were no longer breastfeeding $(8 / 127=6.3 \%)$. These eight participants were excluded from the analyses given that they were not asked the HAPA questions 
at their two week follow-up interview. The final sample consisted of 119 exclusively or partially breastfeeding mothers. Figure 2 displays the sample selection by randomization status to clearly indicate how the final sample was obtained; however, group assignment was not controlled for in these analyses given that there was no overall effect of the intervention on exclusive breastfeeding status at two weeks post partum (Martinez-Brockman, 2017).

\section{[INSERT FIGURE 2 HERE]}

\section{Results}

Descriptive Findings

Participants were on average $27.5(S D=5.4)$ years of age, $23.6(S D=2.9)$ weeks gestation at recruitment, and had an average BMI of $27.4(S D=6.8)$. More than three-quarters self-identified as Hispanic, $68.1 \%$ were single, never married, $71.6 \%$ lived with their partner, and over $60.0 \%$ preferred to speak English. Over $60.0 \%$ had other children at home and 53.5\% had previous breastfeeding experience. The majority of participants (58.4\%) had a month-to-month cell phone plan with unlimited data (85.3\%) and nearly all (96.5\%) owned a Smartphone.

In terms of breastfeeding planning, an equal proportion of participants planned to EBF than planned to partially breastfeed (65.6\%); however, participants planned to EBF for a longer duration [5.4 ( $\mathrm{SD}=5.1)$ months versus $3.5(\mathrm{SD}=4.1)$ months, respectively]. At two weeks post partum, participants reported that their milk came in on average 1.7 ( $\mathrm{SD}=2.2)$ days after giving birth and babies were on average 20.4 days old $(S D=11.5)$ at the time of the two week post partum interview. Other sample characteristics can be found in Table 2, for the full sample and by breastfeeding status.

\section{[INSERT TABLE 2 HERE]}

Factor loadings ranged from 0.54 to 0.99 (Table 3 ) and all indicator variables loaded significantly on their hypothesized constructs $(\mathrm{p}<0.0001)$. With respect to action self-efficacy 
and intentions, measured indicators were included that assessed each construct to three and six months post partum, well beyond the two week follow-up time frame (Table 3). While the means of these measured indicators were smaller, they had the highest factor loadings within the action self-efficacy and intentions constructs, pointing to the effect of long-term planning on actual EBF behavior at two weeks post partum.

\section{[INSERT TABLE 3 HERE]}

\section{Predictors of Exclusive Breastfeeding}

The correlations between latent constructs are presented in Table 4. Correlations were weak or moderate, with a few exceptions. Action self-efficacy and intentions were highly correlated $(r=0.82 ; \mathrm{p}<0.0001)$, as were maintenance and recovery self-efficacy $(r=0.78 ; \mathrm{p}<$ 0.0001). The phase-specific self-efficacy construct correlations ranged from 0.25 to 0.78 . Interestingly, planning was more strongly correlated with maintenance and recovery self-efficacy $(r=0.63$ and 0.49 , respectively) than with $\mathrm{EBF}(r=0.32)$. Planning and recovery self-efficacy were the only variables significantly correlated with EBF at two weeks post partum.

\section{[INSERT TABLE 4 HERE]}

The hypothesized model fit the data well, with CFI $=0.97, \mathrm{TLI}=0.96$, and RMSEA of 0.05 (90\% CI: 0. 03, 0.06; $\mathrm{p}=0.70)$. Figure 3 provides the standardized path coefficients. The model revealed that action self-efficacy (and not outcome expectancies) significantly predicted intention to exclusively breastfeed $(0.80 ; \mathrm{p}<0.001)$. Action self-efficacy also significantly predicted maintenance self-efficacy, measured at time $2(0.32 ; \mathrm{p}=0.001)$. Baseline intentions significantly predicted planning at two weeks post partum $(0.28 ; \mathrm{p}=0.009)$. Planning $(0.55 ; \mathrm{p}=$ 0.006), maintenance self-efficacy $(-0.87 ; p=0.028)$, and recovery self-efficacy $(0.64 ; p=0.026)$ each significantly predicted exclusive breastfeeding behavior. All but one of the proposed relationships were positively associated. For example, higher recovery self-efficacy was related 
to a greater likelihood of exclusive breastfeeding. This did not hold true for maintenance selfefficacy however. In this sample, higher maintenance self-efficacy was related to a lower likelihood of exclusive breastfeeding. The model explained $67.4 \%$ of the variance of intention to exclusively breastfeed, $46.6 \%$ of the variance of planning, $10.2 \%$ of the variance of maintenance self-efficacy and $61.2 \%$ of the variance of recovery self-efficacy.

\section{[INSERT FIGURE 3 HERE]}

Indirect effects were also examined for the three meditational paths in the volitional phase. Planning emerged as a mediator of the effect of maintenance self-efficacy on exclusive breastfeeding $(\hat{\beta}$, indirect effect $=0.31 ; p=0.028)$, and recovery self-efficacy mediated the effect of maintenance self-efficacy on exclusive breastfeeding $(\hat{\beta}=0.50 ; p=0.046)$. Planning did not mediate the effect of intentions on behavior however $(\widehat{\beta}=0.15 ; p=0.053)$.

\section{Discussion}

This study demonstrated the usefulness of the HAPA in explaining the social cognitive predictors of EBF behavior among low-income women who attend WIC. Specifically, maintenance self-efficacy, recovery self-efficacy and planning were shown to be important predictors of EBF status in the post-intentional phase. The path coefficients for both planning and recovery self-efficacy were in the expected direction. By contrast and unexpectedly, women with high maintenance self-efficacy were less likely to be EBF at two weeks post partum. This counterintuitive finding is most likely due to how the maintenance self-efficacy questions were phrased. At two weeks post partum these questions addressed the participant's current breastfeeding status as reported earlier in the interview such that EBF mothers were asked about maintaining their EBF status and partially breastfeeding mothers were asked about maintaining their partial status. For example, for a participant who reported feeding her baby with a 
combination of breast milk and formula, the maintenance self-efficacy questions asked about her confidence in her ability to continue partially breastfeeding. Exclusively breastfeeding participants were asked about the confidence in their ability to continue providing breast milk only. Partially breastfeeding mothers clearly expressed a higher confidence in their ability to continue providing any breast milk, while EBF mothers were not as confident that they could continue exclusively breastfeeding in the face of barriers and setbacks. This explains why the path coefficient was consistently negative in our analyses (i.e., higher maintenance self-efficacy was associated with partial breastfeeding status and lower maintenance self-efficacy with EBF status) and demonstrates the difficulty of maintaining exclusive breastfeeding in the first few weeks of life.

Furthermore, the recovery self-efficacy questions were designed to assess a participant's level of confidence in her ability to resume exclusive breastfeeding. These questions did not address resuming partial breastfeeding since those who were not breastfeeding at all at two weeks were not asked the HAPA questions. This distinction is important when examining the role of recovery self-efficacy as a mediator: the direct effect of maintenance self-efficacy on EBF was negative; however, the indirect effect was positive and significant. A participant's belief in her ability to resume EBF mediated the effect of maintenance self-efficacy on behavior. Ideally, we would have liked to examine breastfeeding status as an effect modifier, assessing the applicability of the HAPA within each group, but our sample size did not allow for this.

Social cognitive factors are the proximal antecedents to breastfeeding behavior and are instrumental in understanding breastfeeding outcomes (de Jager et al., 2013). Structural equation models that exclude these factors may not present a comprehensive picture of the biopsychosocial environment in which women breastfeed. Furthermore, little is understood about 
the post-intentional or volitional factors that affect breastfeeding behavior - the "black box" between intentions and behavior. Other studies that have used SEM to examine the antecedents of breastfeeding behavior did not include post-intentional social cognitive constructs (Dodgson et al., 2003; Lau et al., 2015; Perez-Escamilla et al., 1999; Zhu et al., 2013). In fact, these studies lacked social cognitive constructs altogether, were cross-sectional in nature, and/or were conducted in developing country contexts and thus not applicable to the U.S. WIC population (Lau et al., 2015; Perez-Escamilla et al., 1999; Zhu et al., 2013). Our application of the HAPA model expands the existing literature on the psychosocial predictors of breastfeeding behavior by examining the influence of post-intentional social cognitive factors on EBF, specifically, phasespecific self-efficacy and planning.

This study was unique in that, to our knowledge, it was the first to apply the HAPA model to the design and analysis of a text messaging intervention targeting EBF behavior among low-income WIC participants. Other applications of the HAPA model have shown that planning, maintenance and recovery self-efficacy are significant predictors of health promotion/disease prevention behaviors such as maintaining a healthy diet (Renner et al., 2008; Schwarzer \& Renner, 2000) and engaging in regular breast self-examination (Luszczynska, 2004; Luszczynska \& Schwarzer, 2003). This literature demonstrates that planning is an important intermediary construct that helps to bridge the explanatory gap between intentions and behavior. Oftentimes when a mother intends to breastfeed, she may begin breastfeeding and then stop soon after, or she may never initiate breastfeeding at all. Having specific plans as to "when, where, and how" to breastfeed allows an individual to take concrete steps towards achieving a breastfeeding goal. Our results indicate that planning was an important predictor of EBF, but as a mediator between intentions and behavior, was only borderline significant. 
The role of phase-specific self-efficacy cannot be understated in this process. A breastfeeding woman who is self-efficacious is one who imagines success, is persistent and redoubles her efforts in the face of barriers and setbacks, and responds confidently if breastfeeding plans are derailed (Luszczynska \& Schwarzer, 2003). The LATCH study was designed to enhance each type of phase-specific self-efficacy prenatally and post partum and encouraged participants to develop action and coping plans. Our findings support the role of phase specific self-efficacy in terms of the theoretically predicted relationships between constructs and their influence on planning and EBF behavior.

Breastfeeding peer counseling is a powerful approach to educating and supporting WIC participants who chose to breastfeed. This study demonstrates that text messaging may be a useful tool for BFPCs to enhance the social support they provide to participants and teach and support planning behaviors, thereby augmenting the BFPC experience. Other strengths include the random assignment to study condition, the inclusion of four study sites targeting different geographic and demographic areas of the state, and the single-blind status of the follow-up interviewers.

\section{Limitations}

The study had several limitations. First, our results may not generalizable to WIC populations outside of Connecticut that have a very different ethnographic makeup. Our sample drew from a WIC population that is 51.2\% Hispanic (CT State Department of Public Health, 2015), and thus consisted primarily of Hispanic women. Second, approximately $20 \%$ of the intervention group and $25 \%$ of the control group were lost to follow-up at two weeks postpartum. This may indicate that the mothers who remained in the study were highly motivated to breastfeed and could affect the generalizability of the findings to women without the intention or 
the motivation to breastfeed. Third, the study relied on self-reported breastfeeding status. While this does introduce the potential for reporting bias, self-reported breastfeeding initiation and duration has been found to be both reliable and valid, especially when recalled after a short period (Li et al., 2005). Fourth, the study was underpowered for examining effect modification by breastfeeding modality. Finally, structural equation modeling should be applied to sample sizes $\geq 200$ (Jackson et al., 2009; Schreiber et al., 2006). Because our sample size was below this threshold $(\mathrm{N}=119)$, findings that were not statistically significant should be interpreted with caution to avoid committing a type II error. Despite these limitations, we believe SEM was the best approach to understanding the relationships between the social cognitive predictors in this sample.

\section{Conclusion}

Breastfeeding is a learned skill and the first two weeks post partum represent a crucial period for the establishment of lactation. It is during this time that copious milk production begins, the milk supply begins to be established, and mother and baby learn to breastfeed together. Learning how to resolve breastfeeding issues takes patience and support and mothers build and reinforce their self-efficacy during this process by gaining direct experience and with the help of their support networks. WIC breastfeeding peer counselors provide a crucial form of educational and social support to breastfeeding women during the prenatal, peripartum, and post partum periods. The LATCH intervention presents one innovative approach to augment and reinforce the BFPC process (Harari, 2014). This study demonstrated the successful application of the HAPA model to the design of an mHealth intervention targeting EBF behavior. It also demonstrated the utility of the HAPA model in explaining EBF behavior at two weeks post partum. Future iterations of LATCH should seek expand the content to cover mothers with and 
without the intention to breastfeed and should examine effect modification by breastfeeding modality. 


\section{References}

Anderson, A.K., Damio, G., Young, S., Chapman, D.J., \& Perez-Escamilla, R. (2005). A randomized trial assessing the efficacy of peer counseling on exclusive breastfeeding in a predominantly Latina low-income community. Archives of Pediatrics \& Adolescent Medicine, 159, 836-841.

Bandura, A. (1977). Self-efficacy: toward a unifying theory of behavioral change. Psychology Reviews, 84.

Centers for Disease Control and Prevention. (2014). Infant Feeding Practices Study II (IFPS II). Neonatal questionnaire. Atlanta, GA: CDC.

Centers for Disease Control and Prevention. (2016). Breastfeeding Report Card - United States, 2016. Atlanta, GA.

Chapman, D.J., Damio, G., \& Perez-Escamilla, R. (2004a). Differential response to breastfeeding peer counseling within a low-income, predominantly Latina population. Journal of Human Lactation, 20, 389-396.

Chapman, D.J., Damio, G., Young, S., \& Perez-Escamilla, R. (2004b). Effectiveness of breastfeeding peer counseling in a low-income, predominantly Latina population: a randomized controlled trial. Archives of Pediatrics \& Adolescent Medicine, 158, 897-902.

Chapman, D.J., Morel, K., Anderson, A.K., Damio, G., \& Perez-Escamilla, R. (2010). Breastfeeding peer counseling: from efficacy through scale-up. Journal of Human Lactation, 26, 314-326.

Chapman, D.J., \& Perez-Escamilla, R. (2012). Breastfeeding among minority women: moving from risk factors to interventions. Advances in Nutrition, 3, 95-104. 
Connecticut State Department of Public Health. (2015). State WIC Program Report: 2015 Monthly WIC participation by Race/Ethnicity. Retrieved from http://www.ct.gov/dph/lib/dph/wic_2016/wic_participation_by_race-ethnicity.pdf.

de Jager, E., Skouteris, H., Broadbent, J., Amir, L., \& Mellor, K. (2013). Psychosocial correlates of exclusive breastfeeding: a systematic review. Midwifery, 29, 506-518.

Dodgson, J.E., Henly, S.J., Duckett, L., \& Tarrant, M. (2003). Theory of planned behavior-based models for breastfeeding duration among Hong Kong mothers. Nursing research, 52, $148-158$.

Epstein, C., Collins, A., et al.(2015). WIC Breastfeeding Peer Counseling Study: Phase II follow-up implementation report. Prepared by: Abt Associates. In K. Project Officers: Castellanos-Brown, and Robare, J. (Ed.). Alexandria, VA: USDA, Food and Nutrition Service.

Harari, N., Rosenthal, M.S., Griswold, M., Goeschel, L., Bozzi, V., Fenick, A.M., PerezEscamilla, R. (2014). Impact of a Text Message Intervention Used as an Adjunct Tool by WIC Breastfeeding Peer Counselors: the LATCH Pilot. Pediatric Academic Societies \& Asian Societies for Pediatric Research. Vancouver, B.C.

Healthy People 2020. (2015). Healthy People 2020 Objectives: Maternal, Infant, and Child Health. Retrieved from https://www.healthypeople.gov/2020/topicsobjectives/topic/maternal-infant-and-child-health/objectives

Jackson, D.L., Gillaspy Jr, J.A., \& Purc-Stephenson, R. (2009). Reporting practices in confirmatory factor analysis: an overview and some recommendations. Psychological methods, 14, 6 . 
Jensen, E. (2012). Participation in the Supplemental Nutrition Program for Women, Infants and Children (WIC) and breastfeeding: National, regional, and state level analyses. Maternal and child health journal, 16, 624-631.

Labbok, M., \& Krasovec, K. (1990). Toward consistency in breastfeeding definitions. Stud Fam Plann., 21, 226-230.

Lau, Y., Htun, T.P., Im Lim, P., Ho-Lim, S., \& Klainin-Yobas, P. (2015). Maternal, infant characteristics, breastfeeding techniques, and initiation: Structural equation modeling approaches. PloS one, 10, e0142861.

Li, R., Scanlon, K.S., \& Serdula, M.K. (2005). The validity and reliability of maternal recall of breastfeeding practice. Nutr Rev., 63, 103-110.

Luszczynska, A. (2004). Change in breast self-examination behavior: effects of intervention on enhancing self-efficacy. International Journal of Behavioral Medicine, 11, 95-103.

Luszczynska, A., \& Schwarzer, R. (2003). Planning and self-efficacy in the adoption and maintenance of breast self-examination: A longitudinal study on self-regulatory cognitions. Psychology and Health, 18, 93-108.

Martin, L., Hossain, S., Casanovas, C., \& Guyon, A. (2010). Learning from large-scale community-based programmes to improve breastfeeding practices. In W.H.O. (WHO) (Ed.).

Martinez-Brockman, J.L, Harari, N., Segura-Pérez, S., Goeschel, L., Bozzi, V., Pérez-Escamilla, $R$. (2017). Results of the Lactation Advice Through Texting Can Help (LATCH) Randomized Controlled Trial. Under review, Journal of Nutrition Education \& Behavior.

Michie, S., Richardson, M., Johnston, M., Abraham, C., Francis, J., Hardeman, W., et al. (2013). The Behavior Change Technique Taxonomy (v1) of 93 Hierarchically Clustered 
Techniques: Building an International Consensus for the Reporting of Behavior Change Interventions. Annals of Behavioral Medicine, 46, 81-95.

Muthén, L.K.a.M., B.O. (1998 - 2009). Mplus User's Guide. Los Angeles, CA: Muthén \& Muthén.

Nommsen-Rivers, L.A., Chantry, C.J., Cohen, R.J., \& Dewey, K.G. (2010). Comfort with the idea of formula feeding helps explain ethnic disparity in breastfeeding intentions among expectant first-time mothers. Breastfeeding Medicine: The Official Journal of the Academy of Breastfeeding Medicine, 5, 25-33.

Nommsen-Rivers, L.A., \& Dewey, K.G. (2009). Development and validation of the infant feeding intentions scale. Maternal \& Child Health Journal, 13, 334-342.

Perez-Escamilla, R. (2012). Breastfeeding social marketing: lessons learned from USDA's "Loving Support" campaign. Breastfeeding Medicine: The Official Journal of the Academy of Breastfeeding Medicine, 7, 358-363.

Perez-Escamilla, R., Cobas, J.A., Balcazar, H., \& Holland Benin, M. (1999). Specifying the antecedents of breast-feeding duration in Peru through a structural equation model. Public Health Nutrition, 2, 461-467.

Renner, B., Kwon, S., Yang, B.H., Paik, K.C., Kim, S.H., Roh, S., et al. (2008). Social-cognitive predictors of dietary behaviors in South Korean men and women. International Journal of Behavioral Medicine, 15, 4-13.

Rollins, N.C., Bhandari, N., Hajeebhoy, N., Horton, S., Lutter, C.K., Martines, J.C., et al. (2016). Why invest, and what it will take to improve breastfeeding practices? The Lancet, 387 , 491-504. 
Rothman, A.J., \& Salovey, P. (1997). Shaping perceptions to motivate healthy behavior: the role of message framing. Psychological Bulletin, 121, 3-19.

Ryan, A.S., \& Zhou, W. (2006). Lower breastfeeding rates persist among the Special Supplemental Nutrition Program for Women, Infants, and Children participants, 19782003. Pediatrics, 117, 1136-1146.

Schreiber, J.B., Nora, A., Stage, F.K., Barlow, E.A., \& King, J. (2006). Reporting structural equation modeling and confirmatory factor analysis results: A review. The Journal of educational research, 99, 323-338.

Schwarzer, R. (2008). Modeling health behavior change: How to predict and modify the adoption and maintenance of health behaviors. Applied Psychology: An International Review, 57, 1-29.

Schwarzer, R. (2014). Self-efficacy: Thought control of action: Taylor \& Francis.

Schwarzer, R., \& Renner, B. (2000). Social-cognitive predictors of health behavior: action selfefficacy and coping self-efficacy. Health Psychology, 19, 487-495.

Schwarzer, R., Schuz, B., Ziegelmann, J.P., Lippke, S., Luszczynska, A., \& Scholz, U. (2007). Adoption and maintenance of four health behaviors: theory-guided longitudinal studies on dental flossing, seat belt use, dietary behavior, and physical activity. Annals of Behavioral Medicine, 33, 156-166.

Schwarzer R., L.A. (2008). How to overcome health compromising behaviors. European Psychologist, 13, 141-151.

Tversky, A., \& Kahneman, D. (1981). The framing of decisions and the psychology of choice. Science, 211, 453-458. 
United States Department of Agriculture. (2015). About WIC - WIC at a Glance. In F.a.N.

Service (Ed.). Retrieved from http://www.fns.usda.gov/wic/about-wic-wic-glance

WHO/UNICEF. (2003). Global Strategy for Infant and Young Child Feeding.

World Health Organization. (2015). Exclusive Breastfeeding. e-Library of Evidence for Nutrition Actions (eLENA).

Zhu, P., Hao, J., Jiang, X., Huang, K., \& Tao, F. (2013). New insight into onset of lactation:

mediating the negative effect of multiple perinatal biopsychosocial stress on

breastfeeding duration. Breastfeeding Medicine: The Official Journal of the Academy of

Breastfeeding Medicine, 8, 151-158. 


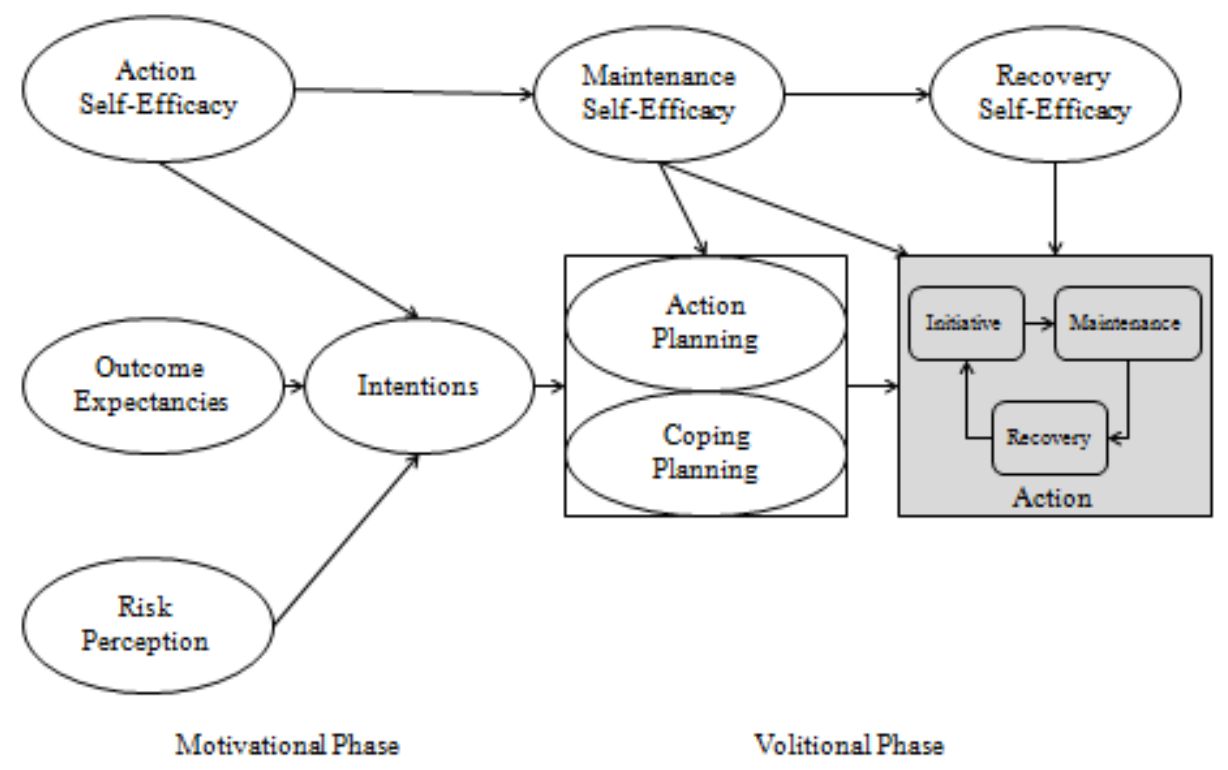

Figure 1. The Health Action Process Approach (Schwarzer, 2008). Notes: Ovals represent each latent social-cognitive construct and arrows indicate the direction of the relationship between latent constructs. In the motivational phase, risk perception, outcome expectancies, and action self-efficacy work together to help an individual form an intention to engage in a health promotion or disease prevention behavior. In the post-intentional or volitional phase, action- and coping-planning bridge the gap between intentions and behavior, while maintenance and recovery self-efficacy play an important role in the planning process as well as the iterative behavioral process. 


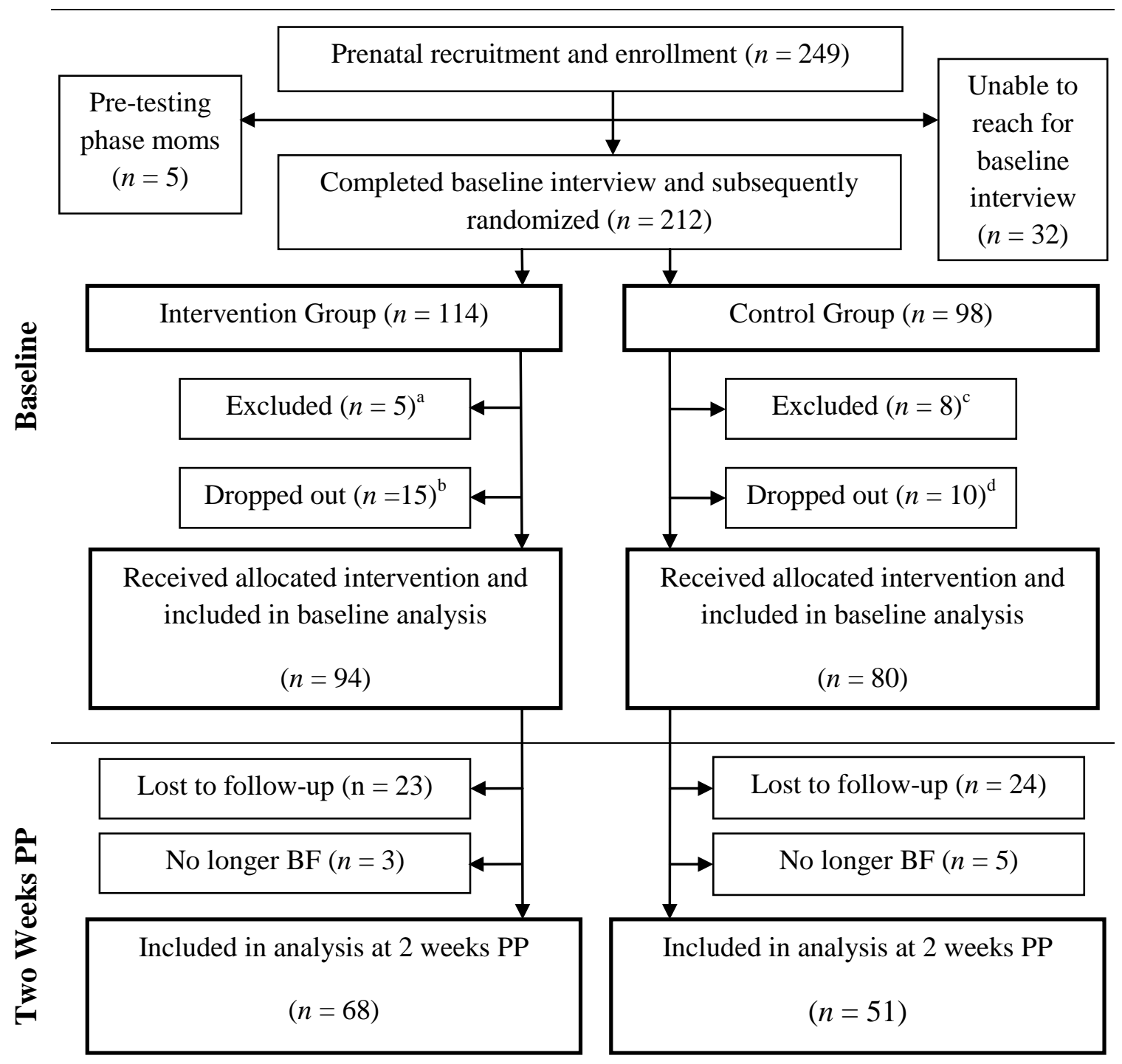

Figure. 2. LATCH Study Design. Figure 2 displays the sample selection process for these analyses.

Notes: ${ }^{\text {a }}$ Reasons for exclusion: premature birth $(n=1)$, withdrew from BFPC $(n=1)$, miscarriage $(n=1)$, not singleton $(n=1),<5$ lbs at birth $(n=1) ;{ }^{\mathbf{b}}$ Reasons for dropping out: opted out of study $(n=9)$, unable to obtain baby's DOB ( $\mathrm{n}=6)$; ${ }^{\mathrm{c}}$ Reasons for exclusion: premature birth $(n=4),<5 \mathrm{lbs}$ at birth $(n=1)$, $>3$ days NICU $(n=1)$, recruited $>28$ weeks gestation $(n=1)$, medication at birth precluded breastfeeding $(n=1) ;{ }^{\mathbf{d}}$ Reasons for dropping out: opted out of study $(n=2)$, unable to obtain baby's DOB $(n=8)$. 


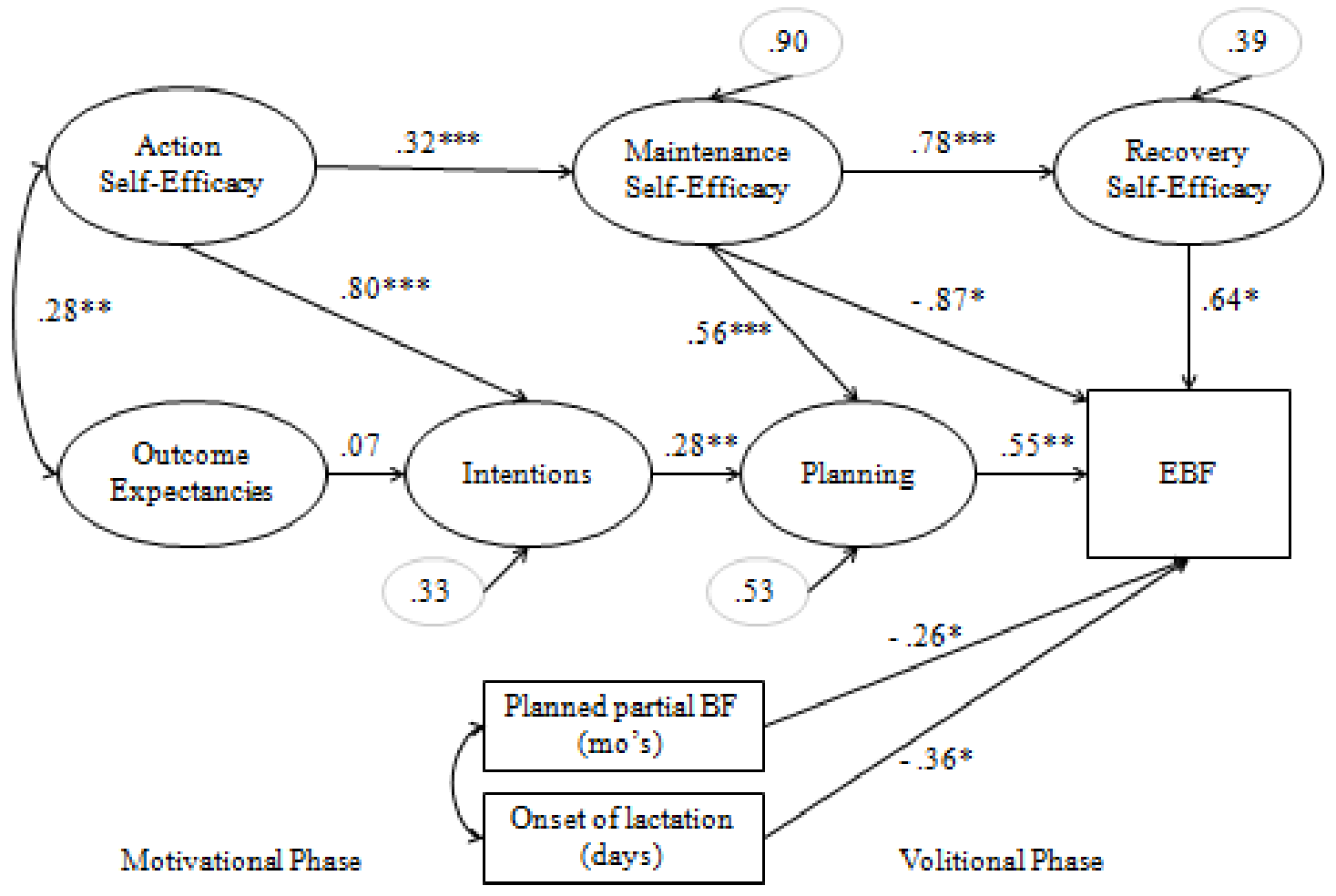

Figure 3. Hypothetical Model with Standardized Regression Coefficients. Ovals represent latent constructs, boxes represent measured variables, and arrows indicate the direction of the relationship. Notes: $* p<0.05 . * * p<0.01 . * * * p \leq 0.001$. 
Table 1

LATCH Message Examples by Period and HAPA Construct ${ }^{\mathrm{a}}$

\begin{tabular}{|c|c|}
\hline \multicolumn{2}{|r|}{ Prenatal } \\
\hline \multicolumn{2}{|l|}{ Action Self-Efficacy } \\
\hline SMS & $\begin{array}{l}\text { No matter what size your breasts are, they will easily make and } \\
\text { supply milk directly in response to your baby's needs. }\end{array}$ \\
\hline MMS & $\begin{array}{l}\text { Some babies latch on right away, but for many it takes more time. } \\
\text { You and your baby will learn together. See video for details: [link] }\end{array}$ \\
\hline \multicolumn{2}{|l|}{ Action Planning } \\
\hline SMS & $\begin{array}{l}\text { Talk to your doctor about how they can help support your plan to } \\
\text { breastfeed. They can help during the first hours and days after birth. }\end{array}$ \\
\hline MMS & $\begin{array}{l}\text { Your baby will love feeling your touch. In the first hour after birth, } \\
\text { let your baby lie on your chest skin to skin. See picture: [link] }\end{array}$ \\
\hline \multicolumn{2}{|r|}{ Post Partum } \\
\hline \multicolumn{2}{|l|}{ Coping Planning } \\
\hline SMS & $\begin{array}{l}\text { To help with milk let-down: try to relax, find a quiet place without } \\
\text { distractions, and focus on your baby. Gentle breast massage can also } \\
\text { help if needed. }\end{array}$ \\
\hline MMS & $\begin{array}{l}\text { Some breastfeeding moms worry about leaking onto their shirt. Feed } \\
\text { baby frequently, before breasts become over full. Leaks are easy to } \\
\text { prevent! For more info: [link] }\end{array}$ \\
\hline \multicolumn{2}{|c|}{ Maintenance Self-Efficacy } \\
\hline SMS & $\begin{array}{l}\text { Your breasts may feel soft and back to normal. Don't worry you are } \\
\text { still making plenty of milk. It means baby is feeding well and } \\
\text { making room for new milk. }\end{array}$ \\
\hline MMS & $\begin{array}{l}\text { Pumping allows you to save breast milk for times you are separated } \\
\text { from your baby. To hear one mom's story, click [link] }\end{array}$ \\
\hline \multicolumn{2}{|l|}{ Recovery Self-Efficacy } \\
\hline SMS & $\begin{array}{l}\text { It is possible to start breastfeeding again, even if you have stopped. } \\
\text { Text me if you have stopped and want help getting started again. }\end{array}$ \\
\hline MMS & $\mathrm{n} / \mathrm{a}$ \\
\hline
\end{tabular}

${ }^{a}$ Abbreviations: SMS = short message service (i.e., text messages only); MMS = multimedia message service (i.e., text and web links to photos, videos or websites all accessed through the mobile web browser). 
Table 2

Sample Characteristics by Breastfeeding Status at 2 Weeks Post Partum $(n=119)^{\mathrm{a}}$

\begin{tabular}{|c|c|c|c|c|}
\hline & \multirow[b]{2}{*}{$\begin{array}{c}\text { Total }^{\mathrm{b}} \\
(n=119)\end{array}$} & \multicolumn{2}{|c|}{ Breastfeeding Status } & \multirow[b]{2}{*}{$p$-value } \\
\hline & & $\begin{array}{c}\mathrm{EBF} \\
(n=61)\end{array}$ & $\begin{array}{c}\text { Partial BF } \\
(n=58)\end{array}$ & \\
\hline \multicolumn{5}{|l|}{ Maternal Biomedical Characteristics } \\
\hline Age (years) & $27.5 \pm 5.4$ & $27.6 \pm 5.3$ & $27.4 \pm 5.6$ & 0.782 \\
\hline Pre-pregnancy BMI $\left(\mathrm{kg} / \mathrm{m}^{2}\right)$ & $27.4 \pm 6.8$ & $26.8 \pm 5.9$ & $28.1 \pm 7.7$ & 0.331 \\
\hline Gestational age at recruit. (wks) & $23.6 \pm 2.9$ & $23.9 \pm 2.9$ & $23.3 \pm 2.8$ & 0.293 \\
\hline Primiparous & & & & 0.432 \\
\hline Yes & $47(39.5)$ & $22(46.8)$ & $25(53.2)$ & \\
\hline No & $72(60.5)$ & $39(54.2)$ & $33(45.8)$ & \\
\hline Onset of lactation (days pp) & $1.7 \pm 2.2$ & $1.2 \pm 1.3$ & $2.2 \pm 2.8$ & 0.015 \\
\hline Baby age (days) & $20.4 \pm 11.5$ & $18.8 \pm 16.2$ & $22.1 \pm 18.8$ & 0.122 \\
\hline \multicolumn{5}{|c|}{ Breastfeeding Planning \& Experience } \\
\hline Planned Any BF duration (mo.) & $9.4 \pm 5.6$ & $9.5 \pm 4.8$ & $9.2 \pm 6.3$ & 0.766 \\
\hline Planned EBF & & & & 0.121 \\
\hline Yes & $78(65.6)$ & $44(56.4)$ & $34(43.6)$ & \\
\hline No & $41(34.5)$ & $17(41.5)$ & $24(58.5)$ & \\
\hline Planned EBF duration (mo.) & $5.4 \pm 5.1$ & $6.0 \pm 4.7$ & $4.8 \pm 5.5$ & 0.225 \\
\hline Planned Partial BF & & & & 0.055 \\
\hline Yes & $78(65.6)$ & $35(44.9)$ & $43(55.1)$ & \\
\hline No & $41(34.5)$ & $26(63.4)$ & $15(36.6)$ & \\
\hline Planned Partial BF duration (mo.) & $3.5 \pm 4.1$ & $2.7 \pm 3.5$ & $4.3 \pm 4.5$ & 0.033 \\
\hline Previous breastfeeding experience & & & & 0.883 \\
\hline Yes & $62(53.5)$ & $33(53.2)$ & $29(46.8)$ & \\
\hline No & $54(46.6)$ & $28(51.9)$ & $26(48.2)$ & \\
\hline \multicolumn{5}{|l|}{ Demographic Characteristics } \\
\hline Ethnicity & & & & 0.636 \\
\hline Hispanic & $90(76.9)$ & $48(53.3)$ & $42(46.7)$ & \\
\hline Non-Hispanic & $27(23.1)$ & $13(48.2)$ & $14(51.9)$ & \\
\hline Marital status & & & & 0.373 \\
\hline Single, never married & $79(68.1)$ & $42(53.2)$ & $37(46.8)$ & \\
\hline Married & $30(25.9)$ & $13(43.3)$ & $17(56.7)$ & \\
\hline Divorced/Separated/Widowed & $7(6.0)$ & $5(71.4)$ & $2(28.6)$ & \\
\hline Living with partner & & & & 0.167 \\
\hline Yes & $83(71.6)$ & $47(56.6)$ & $36(43.4)$ & \\
\hline No & $33(28.5)$ & $14(42.4)$ & $19(57.6)$ & \\
\hline Maternal education & & & & 0.869 \\
\hline High school grad/GED & $33(35.1)$ & $18(54.6)$ & $15(45.5)$ & \\
\hline$<\mathrm{HS}$ & $15(16.0)$ & $9(60.0)$ & $6(40.0)$ & \\
\hline$>\mathrm{HS}$ & $46(48.9)$ & $24(52.2)$ & $22(47.8)$ & \\
\hline Language preference & & & & 0.594 \\
\hline English & $69(60.0)$ & $34(49.3)$ & $35(50.7)$ & \\
\hline Spanish & $46(40.0)$ & $25(54.4)$ & $21(45.7)$ & \\
\hline
\end{tabular}

Cell Phone \& Plan 


\begin{tabular}{|c|c|c|c|c|}
\hline Plan type & & & & 0.738 \\
\hline Month-to-month & $66(58.4)$ & $33(50.0)$ & $33(50.0)$ & \\
\hline Contract & $47(41.6)$ & $25(53.2)$ & $22(46.8)$ & \\
\hline Smartphone & & & & 1.000 \\
\hline Yes & $110(96.5)$ & $58(52.7)$ & $52(47.3)$ & \\
\hline No & $4(3.5)$ & $2(50.0)$ & $2(50.0)$ & \\
\hline Unlimited data & & & & 0.975 \\
\hline Yes & $99(85.3)$ & $52(52.5)$ & $47(47.5)$ & \\
\hline No & $17(14.7)$ & $9(52.9)$ & $8(47.1)$ & \\
\hline \multicolumn{5}{|l|}{ Income \& Food Insecurity } \\
\hline Income & & & & 0.170 \\
\hline$\leq \$ 1,000 / \mathrm{mo}$ & $26(22.2)$ & $13(50.0)$ & $13(50.0)$ & \\
\hline$>\$ 1,000-\$ 2,000+/ \mathrm{mo}$ & $23(19.7)$ & $16(69.6)$ & $7(30.4)$ & \\
\hline Don't know/refused & $68(58.1)$ & $32(47.1)$ & $36(52.9)$ & \\
\hline SNAP & & & & 0.759 \\
\hline Yes & $51(44.0)$ & $26(51.0)$ & $25(49.0)$ & \\
\hline No & $65(56.0)$ & $35(53.9)$ & $30(46.2)$ & \\
\hline
\end{tabular}

Notes: Abbreviations: BMI = Body Mass Index; EBF = exclusive breast feeding; BF = breastfeeding; GED $=$ General Education Development test; SNAP = Supplemental Nutrition Assistance Program.

${ }^{a}$ Table values are mean $\pm S D$ for continuous variables and $n$ (row \%) for categorical variables.

${ }^{\mathbf{b}}$ Numbers may not sum to total due to missing data, and percentages may not sum to $100 \%$ due to rounding.

${ }^{c} P$-value is for 2 -sample $t$-test for normally distributed continuous variables or $\chi^{2}$ test/Fisher's exact test for categorical variables. 
Table 3. Ranges, means, standard deviations and standardized factor loadings of measured indicators.

\begin{tabular}{|c|c|c|c|}
\hline Latent variable/indicator & Range & Mean $(S D)$ & Factor Loading \\
\hline \multicolumn{4}{|l|}{ Outcome expectancies $(\alpha=.85)$} \\
\hline \multicolumn{4}{|c|}{ Do you think if you give your baby breast milk only: } \\
\hline $\begin{array}{l}\text { 1. You will lose weight quickly } \\
\text { after birth? }\end{array}$ & $1-4$ & $3.06(1.02)$ & 0.69 \\
\hline $\begin{array}{l}\text { 2. Your baby will be less likely } \\
\text { to be obese later in life? }\end{array}$ & $1-4$ & $3.10(1.10)$ & 0.82 \\
\hline $\begin{array}{l}\text { 3. It will keep your baby from } \\
\text { getting sick? (e.g., ear infections, } \\
\text { diarrhea) }\end{array}$ & $1-4$ & $3.50(0.88)$ & 0.83 \\
\hline $\begin{array}{l}\text { 4. It will lower your risk of } \\
\text { breast cancer? }\end{array}$ & $1-4$ & $3.12(1.01)$ & 0.89 \\
\hline $\begin{array}{l}\text { 5. It will lower your risk of } \\
\text { diabetes? }\end{array}$ & $1-4$ & $2.81(1.00)$ & 0.90 \\
\hline \multicolumn{4}{|l|}{ Action self-efficacy $(\alpha=.82)$} \\
\hline \multicolumn{4}{|c|}{ Are you sure that you can give your baby breast milk only: } \\
\hline 1. Within 1 hour of giving birth? & $2-4$ & $3.71(0.59)$ & 0.67 \\
\hline $\begin{array}{l}\text { 2. For the first } 24 \text { hours after } \\
\text { giving birth? }\end{array}$ & $2-4$ & $3.77(0.50)$ & 0.70 \\
\hline $\begin{array}{l}3 \text {. For the first } 2 \text { weeks after } \\
\text { your baby is born? }\end{array}$ & $1-4$ & $3.70(0.66)$ & 0.76 \\
\hline $\begin{array}{l}\text { 4. For the first } 3 \text { months after } \\
\text { your baby is born? }\end{array}$ & $1-4$ & $3.50(0.84)$ & 0.99 \\
\hline $\begin{array}{l}5 . \text { For the first } 6 \text { months after } \\
\text { your baby is born? }\end{array}$ & $1-4$ & $3.18(0.96)$ & 0.88 \\
\hline \multicolumn{4}{|l|}{ Intentions $(\alpha=.76)$} \\
\hline 1. I plan to breastfeed my baby & $3-4$ & $3.93(0.25)$ & 0.87 \\
\hline $\begin{array}{l}\text { 2. When my baby is } 2 \text { weeks old, } \\
\text { I will be BF without using } \\
\text { formula or other milk }\end{array}$ & $1-4$ & $3.75(0.63)$ & 0.82 \\
\hline $\begin{array}{l}\text { 3. When my baby is } 3 \text { months } \\
\text { old, I will be BF without using } \\
\text { formula or other milk }\end{array}$ & $1-4$ & $3.36(0.89)$ & 0.95 \\
\hline $\begin{array}{l}\text { 4. When my baby is } 6 \text { months } \\
\text { old, I will be BF without using } \\
\text { formula or other milk }\end{array}$ & $1-4$ & $3.00(1.00)$ & 0.92 \\
\hline \multicolumn{4}{|l|}{ Planning $(\alpha=.82)$} \\
\hline \multicolumn{4}{|l|}{ Do you have a detailed plan of: } \\
\hline $\begin{array}{l}\text { 1. How long you would like to } \\
\text { breastfeed? }\end{array}$ & $1-4$ & $3.54(0.78)$ & 0.63 \\
\hline 2. How you will BF? & $1-4$ & $3.64(0.80)$ & 0.77 \\
\hline $\begin{array}{l}\text { 3. How to get your family and } \\
\text { friends to support your decision }\end{array}$ & $1-4$ & $3.64(0.79)$ & 0.71 \\
\hline
\end{tabular}


to breastfeed?

$\begin{array}{llll}\text { 4. When you will talk to your } & 1-4 & 3.31(1.09) & 0.98\end{array}$

boss about your decision to BF?

5. How to will talk to your boss

about your decision to BF?

6. Who to ask for help if you are $\quad 1-4 \quad 3.80(0.62) \quad 0.69$

having trouble breast feeding?

7. How to check whether your

$\begin{array}{lll}1-4 & 3.54(0.88) & 0.81\end{array}$

baby is getting enough breast

milk?

Maintenance self-efficacy $(\alpha=.72)$

Are you sure you can continue giving your baby breast milk (only) even if:

$\begin{array}{llll}\text { 1. You are given free formula? } & 1-4 & 3.86(0.46) & 0.69\end{array}$

2. Your milk doesn't come in $\quad 1-4 \quad 3.58(0.77) \quad 0.54$

right away?

$\begin{array}{llll}\text { 3. Your baby has trouble } & 1-4 & 3.59(0.73) & 0.70\end{array}$

attaching to your breast right

away?

4. Your family doesn't support $\quad 1-4 \quad 3.84(0.51) \quad 0.89$

your decision to BF?

5. It takes you some time to $\quad 1-4 \quad 3.81(0.53) \quad 0.82$

develop a routine?

Recovery self-efficacy $(\alpha=.76)$

Are you sure you can go back to giving your baby breast milk (only) even:

$\begin{array}{llll}\text { 1. After you have given your } & 1-4 & 3.63(0.73) & 0.73\end{array}$

baby formula? ${ }^{\mathrm{a}}$

2. After you stopped BF because $1-4 \quad 3.38(1.00) \quad 0.90$

it was painful? ${ }^{\mathrm{b}}$

3. If you are unsure if your baby $\quad 1-4 \quad 3.26(0.95) \quad 0.86$ is getting enough milk? ${ }^{\mathrm{b}}$

${ }^{a} n=62$. Participants who were partially breastfeeding at 2 weeks post partum were not asked this question; instead they were asked: "Earlier you mentioned you are feeding your baby with a combination of breast milk and formula. Would you like to go back to giving your baby breast milk only? Yes (1), or No (2). If no, then they were not asked the RSE questions.

${ }^{\mathrm{b}} n=108$. Ten participants were missing data for this question. 
Table 4

Correlations Between Latent Constructs ${ }^{\mathrm{a}}$

\begin{tabular}{lccccccc}
\hline & $\mathbf{1}$ & $\mathbf{2}$ & $\mathbf{3}$ & $\mathbf{4}$ & $\mathbf{5}$ & $\mathbf{6}$ & $\mathbf{7}$ \\
\hline 1. Outcome expectancies & 1.00 & & & & & & \\
2. Action self-efficacy & 0.28 & 1.00 & & & & & \\
3. Intentions & 0.29 & 0.82 & 1.00 & & & & \\
4. Planning & 0.13 & 0.40 & 0.42 & 1.00 & & & \\
5. Maintenance self-efficacy & 0.09 & 0.32 & 0.26 & 0.63 & 1.00 & & \\
6. Recovery self-efficacy & 0.07 & 0.25 & 0.21 & 0.49 & 0.78 & 1.00 & \\
7. EBF at 2 weeks & $0.04^{\mathrm{ns}}$ & $0.10^{\mathrm{ns}}$ & $0.13^{\mathrm{ns}}$ & 0.32 & $-0.03^{\mathrm{ns}}$ & 0.22 & 1.00 \\
\hline Note: & a EBF = Exclusive Breastfeeding; all $p$ s $<0.05$ except where indicated; ${ }^{\mathrm{ns}} p>0.05$.
\end{tabular}

